\title{
El vínculo entre los controles migratorios y la desigualdad global
}

\author{
The relationship between immigration \\ controls and global inequality
}

Helen Durgante*

Resumen. Este trabajo tiene como objetivo destacar de manera breve algunas violaciones de derechos humanos fundamentales derivadas del control de la inmigración a nivel global, sustentadas en las leyes de inmigración para el control social, económico y político de la población. Así, se hace hincapié en el papel de las políticas neoliberales que garantizan la criminalización de los inmigrantes indocumentados, las estrategias políticas que aumentan la percepción de patriotismo, la explotación de la fuerza de trabajo como neocolonialismo y, por último, los intentos disfrazados de acuerdos de zonas sin fronteras (TLCAN) en el marco del sistema capitalista, como un intento de mejorar la movilidad dentro de los países miembros. Las perspectivas de control de fronteras la consideran como una poderosa herramienta moderna para la dominación y la subordinación de la movilidad geográfica de las personas puesto que mantiene a una elite en el control y como resultado persiste la desigualdad, en última instancia, a nivel mundial.

Palabras clave. derechos humanos, migración, políticas neoliberales, indocumentados, capitalismo.

\begin{abstract}
This paper aims to briefly stress some fundamental human rights violations derived from immigration control at the global level, based on immigration laws for social, economic and political control of population. In that, it emphasizes the role of neo-liberal policies to ensure the criminalization of undocumented immigrants, political strategies to increase perceptions of patriotism, exploitation of labor force as neo colonialism and finally, the disguised attempts of borderless zones (NAFTA) under the capitalistic system, presumably as an attempt to enhance mobility within member countries. Perspectives of border control are drawn as a modern powerful tool for dominance and subordination of people's geographic movement in order to maintain elite control and, as a result, ultimate global inequality.
\end{abstract}

Keywords. human rights, immigration, neo-liberal policies, undocumented, capitalistic system. 



\section{Introducción}

El nuevo sistema nacional de control migratorio, visas y regulaciones afines, fue implementado de modo decisivo a principios del siglo XX (Martin, 2003). Esto permitió que los gobiernos tuvieran un mayor control sobre el movimiento de la gente por el mundo, lo que a su vez implicó un mayor poder socioeconómico y político, ya que los Estados fueron capaces de manejar información, flujo del capital y fuerza laboral (Bornstein, 2002). A pesar de ello, últimamente la legalidad del control fronterizo ha sido cuestionada en términos de sus posibles efectos perniciosos, de las restricciones de los derechos de los individuos y de los movimientos estacionales de los trabajadores, un fenómeno global relativamente nuevo que cambia de acuerdo con el movimiento del capital. Más aún, incluso en el caso de trabajadores migrantes restringidos mediante las reglas impuestas por la elite, se tiende a controlar el flujo geográfico del capital y en consecuencia se definen nuevas formas para los sitios de producción y de desigualdad (Hart, 2006). El objetivo de este artículo es destacar con concisión algunas violaciones a derechos humanos fundamentales que resultan del control migratorio a escala

global y que se basan en leyes de inmigración para el control social, económico y político de la población.

De manera reciente, el control migratorio ha sido entendido como una nueva forma de apartheid ${ }^{1}$ global o como estructuras intrínsecas cuyo propósito es garantizar el funcionamiento de sociedades basadas en la administración formal de leyes y sistemas nacionales para la exclusión-inclusión transnacional de individuos en territorios específicos (Hart, 2006). En tal sentido, se planteó que la preeminencia del control migratorio era el medio fundamental para mantener las leyes y el funcionamiento del Estado, al asumir que la coexistencia de inmigrantes ilegales en cualquier área tiende a la contradicción de las políticas porque transgrede el «orden general» e impacta en la identidad nacional.

Desde esa perspectiva, el control estatal geográfico de los desplazamientos ayudaría a asegurar el desarrollo de un orden nacional categórico para el control estatal de la población. De acuerdo con William (1990, en Grupta, 1994), el

${ }^{1}$ Nota del traductor: No existe una traducción exacta del término apartheid en tanto describe una situación particular de segregación. Algunos términos similares son: Ghetto y enclave. Sin embargo, la idea que se desarrolla hace énfasis en una escala global que en cierta forma sugiere la menor importancia de la localización espacial y destaca más bien la mayor importancia de la exclusión. 
nacionalismo sería un fenómeno social moderno que resulta de la imaginación política de una sociedad homogénea, la cual implica una conciencia nacional, valores y principios para una identificación colectiva basada en la construcción territorial de los vínculos asociativos y en las barreras nacionales, situación que exacerba las diferencias entre comunidades.

En el mismo sentido, los críticos de la división del territorio observan que al destacar abiertamente las diferencias, como opuestas a las similitudes entre naciones, los individuos estarían más proclives a experimentar vulnerabilidad y falta de acceso a sus derechos. Por cierto, las disposiciones administrativas de las fronteras han sido muy criticadas pues promueven violaciones de derechos humanos, en tanto que las personas pueden ser calificadas como apátridas sin capacidad de ejercer sus derechos soberanos. En efecto, esto ha sido observado en particular en el antiguo Centro de Inmigrantes de Sangatte en Calais, Francia (1999, en Fassin, 2005), que era supuestamente un campo de tránsito o de alojamiento temporal para migrantes o solicitantes de asilo en su ruta a Inglaterra. Según el Convenio de Inmigración y Asilo² (1999, en Good, 2004:114), un solicitante de asilo es alguien que «debido a fundado temor de ser perseguido por razones de raza, religión, nacionalidad, pertenencia a un grupo social particular u opinión política, está fuera del país de su nacionalidad y es incapaz de o, debido a tal temor, no desea aceptar para sí la protección de tal país».

En ese caso, la paradoja del control fronterizo para la provisión de ayuda humanitaria a los inmigrantes fue más clara cuando el gobierno británico redujo los permisos de inmigración en un intento por reducir las entradas ilegales a través de las fronteras, lo que resultó en la acumulación de migrantes en Sangatte (cerca de mil 500 personas en vez de las doscientos o trescientos inicialmente previstas). No es necesario decir que los inmigrantes en el Centro Sangatte no podían reclamar soberanía nacional de ningún lado, ni de los británicos ni de los franceses.

Además, la Cruz Roja Francesa reportó las precarias condiciones del campo como similares a las de los confinamientos o campos de concentración en la Segunda Guerra Mundial. Allí, la gente no podía satisfacer sus necesidades humanas básicas más que con lo que voluntariamente había sido donado y

${ }^{2}$ Nota del traductor: En materia jurídica (derecho internacional), el término act podría — dependiendo del caso- tener connotaciones particulares, por lo que su traducción como acta, tratado o convenio, por ejemplo, podrían no ser adecuados. Aunque se ha sugerido el término convenio, sería preferible mantener su denominación en el idioma original. 
destinado para la provisión de comida y asistencia médica. Es importante comentar que la similitud de Sangatte con los campos de concentración llamó la atención de la comunidad internacional, hecho que influyó la percepción del público acerca de las «inhumanas» condiciones del campo y asimismo respecto de las políticas y las medidas restrictivas gubernamentales. Para colmo de males, el Campo Sangatte fue cerrado en 2002 por el nuevo gobierno de derecha que argumentó que el campo atraería inmigrantes ilegales a la zona y que representaba una amenaza al orden público basado en estructuras democráticas en una sociedad moderna. En otras palabras, el campo fue considerado como una vergüenza nacional y los solicitantes de asilo fueron de nuevo desplazados internamente y se convirtieron en apátridas sin ciudadanía ni reconocimiento gubernamental (Fassin, 2005).

Cabe resaltar también que las ideas contextualizadas de espacios territoriales y límites fueron consideradas en términos de su capacidad de acentuar el sentimiento patriótico y el vínculo nacionalista de los individuos para que los constructos políticos y sociales de las diferentes categorías de individuos fuesen aceptadas por el público en general, facilitando por tanto la manipulación estatal de los flujos de capital y reduciendo el flujo de personas como una condición para la industrialización (Escobar et al., 2003). A partir de lo anterior se establecieron "pseudofronteras», o incluso divisiones ideológicas en función de las nacionalidades, tal vez para conveniencia del Estado nación y por razones económicas, en particular con el propósito de establecer negocios con países del tercer mundo que por lo general son productores de materias primas e importadores de bienes industrializados. Aquí, las fronteras pueden actuar como herramientas fundamentales dentro del marco legal de "comunidades imaginarias», lo que subsecuentemente permite el control capitalista de la fuerza de trabajo. Es interesante notar que esto ha sido ilustrado en el contexto del México rural (exacerbado desde la década de los 1980), donde la dependencia de los campesinos respecto de la migración a Estados Unidos en busca de trabajo pareció derivar en déficits aún mayores, contrario al mejoramiento que se esperaba de las condiciones de vida mediante las remesas de dólares americanos enviadas a sus países de origen (Bindford, 2003).

Durand et al. (1996, en Bindford, 2003) sostuvieron que una mayor desigualdad podría ser el resultado de las remesas en dólares de inmigrantes mexicanos mal pagados. En última instancia los beneficiarios indirectos son en realidad los agronegocios capitalistas de Estados Unidos, los cuales mantienen los medios 
pertinentes para multiplicar y acumular el capital, al propiciar una dependencia superior del trabajo estacional de los mexicanos en Estados Unidos y convertirse en un círculo vicioso. Además se descubrió que de manera general solamente una pequeña parte de los ingresos de los inmigrantes económicos se remite a su país de origen, mientras que el monto restante se queda en el país de origen para cubrir las condiciones de vida de los migrantes; o incluso se descuenta por las compañías capitalistas de envío de remesas. Todo ello representa una doble ganancia de los países industrializados que se benefician de la capacidad laboral barata de los inmigrantes (Blindford, 2003).

A partir de lo expuesto, y bajo el actual sistema capitalista/neoliberal, es posible argumentar que la ilegalidad vinculada a la raza o afiliación étnica fue convenientemente criminalizada en beneficio de las naciones industrializadas y las subsecuentes implicaciones de ser «estatuas» indocumentadas en el Estado nación, tal como la creciente vulnerabilidad de la fuerza laboral barata, restricciones en el acceso a servicios públicos, amenazas frecuentes de deportación y así sucesivamente, lo cual puede ser el resultado de prácticas estandarizadas de control migratorio (Bornstein, 2002). Así, se ha considerado que el poder gubernamental juega un papel clave en la recreación/reproducción de las categorías «ciudadano»y «no-ciudadano». En correspondencia con ello y sobre esa base la autoridad del Estado asegura el control fronterizo y se beneficia de la explotación de la capacidad laboral barata de los inmigrantes, circunstancia que constituye una de las principales razones subyacentes de la desigualdad global.

Según Hamilton y Whaley (1984, en Martin, 2003), si se eliminara el control fronterizo de la inmigración, el PIB global (considerando el Producto Interno Bruto como un índice del nivel de vida de los países) podría duplicarse en los países más pobres. De hecho, la evidencia contextual de Palestina sugiere que el control de la frontera israelí-palestina es en la actualidad uno de los factores primordiales que contribuye con su subdesarrollo. En otras palabras, las medidas restrictivas para la importación/exportación, las condiciones extremas y riesgosas que enfrentan los trabajadores palestinos tras cruzar la frontera con Israel (Línea Verde), el acceso a servicios de salud y educación, la inexistencia o la limitación de la compensación laboral y los salarios considerablemente menores comparados con los de los trabajadores israelíes, comprenden aspectos que permiten afirmar que el control fronterizo y las restricciones reflejan un patrón neocolonial, en el que la subordinación y la explotación de una cierta región asegura el desarrollo y el progreso de otra región dominante (Bornstein, 2002). 
De la misma importancia es el acceso negociado a las fronteras políticas y sociales que hoy se maneja únicamente a través de regulaciones hechas por el Estado (Graeber, 1995). Al respecto, los derechos de desplazamiento de los individuos, la estratificación social, el asilo y la extensión de visas, por ejemplo, se hallan sujetos a ordenamientos jerárquicos para el control estratégico del Estado sustentados en leyes de inmigración que definen quienes serán «subhumanos» o miembros extranjeros de una determinada sociedad; lo cual puede variar de un país a otro según las maneras en que se experimenta la migración. En consecuencia, se reconfigura la percepción general de la disponibilidad/deportabilidad de alguien que no es ciudadano (Escobar et al., 2003). En efecto, varias investigaciones han estimado que el costo de ser un inmigrante indocumentado se encuentra entre los más elevados en lo concerniente a las violaciones a los derechos humanos; donde se supone que los grupos vulnerables experimentan varias penurias emocionales, psicológicas y físicas, debido a la soledad, la explotación, la discriminación/marginación y los abusos de todo tipo. Cuando los individuos no pueden cumplir las exigencias impuestas ni las directrices del sistema actual se les niega el acceso a sus derechos (Mahler, 1995, en Blindford, 2003).

Además, resalta una interpretación interesante que plantea la existencia de un intento neoliberal para reducir las barreras entre países miembros, por ejemplo en el caso del Tratado de Libre Comercio de América del Norte (TLCAN) (Massey, Durand y Malone, 2003). Dicho tratado fue promovido como un acuerdo de integración a largo plazo entre sus Estados miembros (Canadá, Estados Unidos y México) con la finalidad de expandir el margen fronterizo, facilitar así el flujo internacional de capital y posiblemente reducir la desigualdad social. Sin embargo, el efecto del acuerdo, relacionado con la minimización de aranceles restrictivos para operaciones comerciales sin reajustar las políticas que promuevan la movilidad geográfica de las personas entre países, hizo que los pequeños campesinos en México se quedaran sin medios para competir contra las grandes corporaciones subsidiadas de Estados Unidos.

En ese sentido, se especuló que la implementación del TLCAN comprendía un obstáculo para la migración laboral estacional indocumentada a Estados Unidos (braceros). De manera notable, se mostró que el número de guardias fronterizos en Estados Unidos se triplicó desde la implementación del TLCAN, cuyos resultados fueron el libre flujo de capitales y bienes, por un lado; y la afectación significativa del flujo de personas entre los países, por el otro (Graeber, 1995). Desde ese punto de vista, lo que presumiblemente fue un intento diseñado para 
dar cuenta de la desigualdad social y mejorar la integración económica y el desarrollo al interior y entre países, en realidad resultó en implicaciones sociales mayores para las comunidades rurales mexicanas.

En síntesis, se considera que se ha producido una nueva tendencia de segregación, que se encuentra en correspondencia con el flujo de capitales manejado por una elite establecida gracias al apoyo de la política internacional y garantizada por el control fronterizo. Asimismo, al implementar globalmente los sistemas de control fronterizo, los grupos sociales más pobres quedan con pocos medios para tratar de mitigar su falta de acceso social, económico y político o emigrar hacia áreas geográficas alternativas en busca de oportunidades. Los Estados pueden beneficiarse del mantenimiento estratégico del control de fronteras al mejorar la percepción de una identidad nacionalista/patriótica, dado que la idea de los «inmigrantes» amenaza el orden social al representar una noción vergonzosa para una sociedad capitalista moderna. Adicionalmente, los inmigrantes indocumentados siguen siendo uno de los grupos más vulnerables a nivel global, al tener que adaptarse en muchos casos a condiciones inhumanas, en su objetivo de llegar o permanecer en el lugar de destino elegido. Se han mencionado algunas especulaciones acerca de la función del control fronterizo como un patrón neocolonial que asegura que la elite tenga el control de áreas subordinadas, por medio de la negación de los derechos soberanos de los individuos.

Finalmente, los tratados sin fronteras ficticios (como el TLCAN), que en esencia se fundamentan en estructuras capitalistas para la industrialización o para incrementar los flujos de capital y controlar las fronteras entre los países miembros, se han propuesto como una alternativa en el manejo de la desigualdad social. No es necesario reiterar la paradoja de la mencionada estrategia que provocó niveles superiores de desigualdad en áreas menos capaces de competir contra grandes corporaciones subsidiadas. Por tanto, las aparentes consecuencias del control de fronteras resultan ser un impedimento para la igualdad social y los derechos humanos en el mundo, puesto que la creación e imposición de Estados enfatizan las diferencias entre las naciones en oposición al reconocimiento de sus similitudes. A través de sus efectos, se podrían explicar las caóticas etapas de medidas preventivas contra los «intrusos», los estados globales de alerta por miedo a perder la propia identidad nacional, o inclusive las amenazas de «escasez» (de recursos) debido a la presencia de inmigrantes en una determinada sociedad. 


\section{Referencias}

Bindford, Leigh (2003), "Migrant remittances and (under) development of Mexico", Critique of Anthropology (23), pp. 305-336.

Bornstein, Avram (2002), "Borders and the utility of violence: State effects on the «superexploitation» of West Bank Palestinians», Critique of Anthropology (22), pp. 201-220.

Escobar, Agustin, Philip Martin, Peter Schatzer \& Ssusan Martin (2003), «Mexico-US migration: Moving the agenda forward», International Migration (41), pp. 125-137.

Fassin, Didier (2005), «Compassion and repression: The moral economy of immigration policies in France», Cultural Anthropology (20), pp. 362-387.

Graeber, David (1995), "The Globalization Movement: Some Points of Clarification», in Edelman, Marc \& Angelique Haugerud (eds.), The Anthropology of development and globalization. From classical political economy to contemporary neoliberalism, New York, Blackwell.

Good, Anthony (2004), "Undoubtedly an expert? Anthropologists in British Asylum Courts», Journal of the Royal Anthropological Institute (10), pp. 113-133.

Grupta, Akhil (1994), «The Song of the nonaligned world. Transnational identities and the reinscription of space in late capitalism», Cultural Anthropology (7), pp. 63-79.

Hart, Keith (2006), "Globalization and apartheid», At the Memory Bank (4), pp. 1-9.

Immigration and Asylum Act (1999), "Chapter 33 Arrangement of Sections», UK, The Stationery Oce Limited.

Martin, Philip (2003), «Economic integration and migration: The Mexico-US case», World Institute for Development Economics Research (35), pp. 1-18.

Massey, Douglas, Jorge Durand \& Nolan Malone (2003), Beyond smoke and mirrors: Mexican immigration in an era of economic integration, New York, Russell Sage Foundation. 